Publ. RIMS, Kyoto Univ.

18 (1982), 427-438

\title{
Semantical Analysis of Constructive PDL
}

\author{
By \\ Hirokazu NISHIMURA*
}

\section{§. Introduction}

Propositional dynamic logic or PDL is an interesting arena of logical research which was born to modal logic as his father and verification logic in the tradition of Floyd/Hoare as his mother. Several completeness proofs of PDL have been presented and the most recent one is Leivant's [4], where constructive or intuitionistic PDL (simply CPDL) plays an auxiliary role. The main purpose of this paper is to give a semantical analysis of CPDL after the manner of Nishimura [5]. In Section 2 we give a Kripkian semantics to CPDL, with respect to which the semantical completeness of a Gentzen-style system introduced in Section 3 is established in Section 4. A secondary purpose of the paper is to show that the existence of a test program A ? does not make our completeness proof so tedious, contrary to Leivant's remarks.

\section{$\S$ 2. Formal Language and Semantics}

There are letters $a_{i}$ and $p_{i}(i=0,1,2, \cdots)$ for atomic programs and propositions respectively, for which we use $a, b, \cdots$ and $p, q, \cdots$ as syntactic variables. We define the notions of a formula and a program by simultaneous induction as follows:

(1) Each atomic proposition $p$ is a formula.

(2) If $A$ and $B$ are formulae, so are $A \wedge B, A \vee B, \neg A$ and $A \supset B$.

(3) If $\alpha$ is a program and $A$ is a formula, then $[\alpha] A$ is a formula.

(4) Each atomic program $a$ is a program.

Received July 8, 1981.

* Research Institute for Mathematical Sciences, Kyoto University, Kyoto 606, Japan. 
(5) If $\alpha$ and $\beta$ are programs, so are

$$
\alpha ; \beta, \quad \alpha \cup \beta \text { and } \alpha^{*} \text {. }
$$

(6) If $A$ is a formula, then $\mathrm{A}$ ? is a program.

true is an abbreviation of $p_{0} \supset p_{0}$. We define $\alpha^{n}$ by induction on $n$; $\alpha^{1}=\alpha$ and $\alpha^{n+1}=\alpha^{n} ; \alpha$.

A sequent is an ordered pair $(\Gamma, \Delta)$ of finite sets of formulae, which we usually denote by $\Gamma \rightarrow \Delta$.

A structure is of the form $(S, \leqq, \rho, \pi)$, where

(1) $S$ is a nonempty set;

(2) $\leqq$ is a partial order on $S$;

(3) $\rho$ is a function assigning to each atomic program a binary relation $\rho(a)$ such that $t \leqq s$ and $\left(s, s^{\prime}\right) \in \rho(a)$ imply $\left(t, s^{\prime}\right) \in \rho(a)$ for any $s, s^{\prime}, t \in S$;

(4) $\pi$ is a function assigning a value in $\{0,1\}$ to each pair $(t, p)$, where $t \in S$ and $p$ is an atomic proposition, such that $\pi(s, p)=1$ and $s \leqq s^{\prime}$ imply $\pi\left(s^{\prime}, p\right)=1$ for any $s, s^{\prime} \in S$.

$\rho$ and $\pi$ are extended to all programs and formulae by simultaneous induction as follows:

(1) $\rho(\alpha ; \beta)=\rho(\alpha) \circ \rho(\beta)$ (composition).

(2) $\rho(\alpha \cup \beta)=\rho(\alpha) \cup \rho(\beta)$ (union):

(3) $\rho\left(\alpha^{*}\right)=\rho$ (true?) $\cup \rho(\alpha) \cup \rho\left(\alpha^{2}\right) \cup \rho\left(\alpha^{3}\right) \cup \cdots$ (iteration).

(4) $\rho(A$ ? $)=\{(s, t) \in S \times S \mid s \leqq t$ and $\pi(t, A)=1\}$.

(5) $\pi(t, A \wedge B)=1$ iff $\pi(t, A)=1$ and $\pi(t, B)=1$.

(6) $\pi(t, A \bigvee B)=1$ iff $\pi(t, A)=1$ or $\pi(t, B)=1$.

(7) $\pi(t, \neg A)=1$ iff for all $s \in S, t \leqq s$ implies $\pi(s, A)=0$.

(8) $\pi(t, A \supset B)=1$ iff for all $s \in S, t \leqq s$ and $\pi(s, A)=1$ imply $\pi(s, B)$ $=1$.

(9) $\pi(t,[\alpha] A)=1$ iff for any $s \in S,(t, s) \in \rho(\alpha)$ implies $\pi(s, A)=1$.

We can readily see the following proposition. 
Proposition 2.1. For any program $\alpha$ and any formula $A$, we have that:

(1) $t \leqq s$ and $\left(s, s^{\prime}\right) \in \rho(\alpha)$ imply $\left(t, s^{\prime}\right) \in \rho(\alpha)$ for any $s, s^{\prime}, t \in S$.

(2) $t \leqq s$ and $\pi(t, A)=1$ imply $\pi(s, A)=1$.

Proof. By induction on $\alpha$ or $A$.

Our syntax is slightly redundant because $A \supset B$ can be regarded as an abbreviation of $[A$ ?] $B$ and similarly for $7 A$. However we do not necessarily prefer to get rid of this redundancy because several subsystems of our syntax (e.g., a test-free variant) are of interest.

A sequent $\Gamma \rightarrow \Delta$ is called realizable if for some structure $(S, \leqq$, $\rho, \pi)$ and some $t \in S$, we have that:

(1) $\pi(t, A)=1$ for any $A \in \Gamma$.

(2) $\pi(t, B)=0$ for any $B \in \Delta$.

A sequent $\Gamma \rightarrow \Delta$ which is not realizable is called valid (notation: $\models \Gamma \rightarrow \Delta)$.

\section{§ 3. Formal System}

Our formal system $\mathbf{L J P}$ for CPDL consists of the following axioms and inference rules:

Axioms: $\quad A \rightarrow A$

Rules: $\quad \frac{\Gamma \rightarrow \Delta}{\Pi, \Gamma \rightarrow \Delta, \Sigma}$ (extension)

$$
\begin{array}{ll}
\frac{\Gamma \rightarrow \Delta, A \quad A, \Pi \rightarrow \Sigma}{\Gamma, \Pi \rightarrow \Delta, \Sigma} & \text { (cut) } \\
\frac{\Gamma \rightarrow \Delta, A \quad \Gamma \rightarrow \Delta, B}{\Gamma \rightarrow \Delta, A \wedge B} & (\rightarrow \wedge)
\end{array}
$$

$$
\left.\begin{array}{c}
\frac{A, \Gamma \rightarrow \Delta}{A \wedge B, \Gamma \rightarrow \Delta} \\
\frac{B, \Gamma \rightarrow \Delta}{A \wedge B, \Gamma \rightarrow \Delta}
\end{array}\right\} \quad(\wedge \rightarrow)
$$




$$
\begin{aligned}
& \left.\begin{array}{c}
\frac{\Gamma \rightarrow \Delta, A}{\Gamma \rightarrow \Delta, A \bigvee B} \\
\frac{\Gamma \rightarrow \Delta, B}{\Gamma \rightarrow \Delta, A \bigvee B}
\end{array}\right\}(\rightarrow \bigvee) \\
& \frac{A, \Gamma \rightarrow \Delta \quad B, \Gamma \rightarrow \Delta}{A \bigvee B, \Gamma \rightarrow \Delta}(\bigvee \rightarrow) \\
& \underset{\Gamma \rightarrow \Delta,\urcorner A}{A, \Gamma \rightarrow}(\rightarrow\urcorner) \\
& \frac{\Gamma \rightarrow \Delta, A}{7 A, \Gamma \rightarrow \Delta}(\neg \rightarrow) \\
& \frac{A, \Gamma \rightarrow B}{\Gamma \rightarrow \Delta, A \supset B} \quad(\rightarrow \supset) \\
& \frac{\Gamma \rightarrow \Delta, A \quad B, \Pi \rightarrow \Sigma}{A \supset B, \Gamma, \Pi \rightarrow \Delta, \Sigma}(\supset \rightarrow) \\
& \frac{\Gamma \rightarrow A}{[\alpha] \Gamma \rightarrow[\alpha] A} \quad(\rightarrow[]) \\
& \frac{\Gamma \rightarrow \Delta,[\alpha][\beta] A}{\Gamma \rightarrow \Delta,[\alpha ; \beta] A} \quad(\rightarrow[;]) \\
& \frac{[\alpha][\beta] A, \Gamma \rightarrow \Delta}{[\alpha ; \beta] A, \Gamma \rightarrow \Delta} \quad([;] \rightarrow) \\
& \frac{\Gamma \rightarrow \Delta,[\alpha] A \quad \Gamma \rightarrow \Delta,[\beta] A}{\Gamma \rightarrow \Delta,[\alpha \cup \beta] A}(\rightarrow[\cup]) \\
& \left.\begin{array}{c}
\frac{[\alpha] A, \Gamma \rightarrow \Delta}{[\alpha \cup \beta] A, \Gamma \rightarrow \Delta} \\
\frac{[\beta] A, \Gamma \rightarrow \Delta}{[\alpha \cup \beta] A, \Gamma \rightarrow \Delta}
\end{array}\right\} \\
& \frac{A \rightarrow[\alpha] A}{A \rightarrow\left[\alpha^{*}\right] A} \quad(\rightarrow[*]) \\
& \left.\begin{array}{l}
\frac{A, \Gamma \rightarrow \Delta}{\left[\alpha^{*}\right] A, \Gamma \rightarrow \Delta} \\
\frac{[\alpha]\left[\alpha^{*}\right] A, \Gamma \rightarrow \Delta}{\left[\alpha^{*}\right] A, \Gamma \rightarrow \Delta}
\end{array}\right\} \quad([*] \rightarrow)
\end{aligned}
$$




$$
\begin{aligned}
& \frac{A, \Gamma \rightarrow B}{\Gamma \rightarrow \Delta,[A ?] B} \quad(\rightarrow[?]) \\
& \frac{\Gamma \rightarrow \Delta, A \quad B, \Pi \rightarrow \Sigma}{[A ?] B, \Gamma, \Pi \rightarrow \Delta, \Sigma} \quad([?] \rightarrow)
\end{aligned}
$$

A proof $P$ (in $\mathbf{L J P}$ ) is a tree of sequents satisfying the following conditions:

(1) The topmost sequents of $P$ are axiom sequents.

(2) Every sequent in $P$ except the lowest one is an upper sequent of an inference rule whose lower sequent is also in $P$.

A sequent $\Gamma \rightarrow \Delta$ is said to be provable (in $\mathbb{L J P}$ ) if there exists a proof whose lowest sequent is $\Gamma \rightarrow \Delta$. If a sequent $\Gamma \rightarrow \Delta$ is provable, then we write $\vdash \Gamma \rightarrow \Delta$ (in $\mathbb{L J P}$ ). A sequent $\Gamma \rightarrow \Delta$ which is not provable is said to be consistent (in $\mathbf{L} \boldsymbol{J P}$ ). A sequent $\Gamma \rightarrow \Delta$ is called intuitionistic if $\Delta$ consists of at most one formula. We denote by $\mathbf{L} J \mathbb{P}^{\prime}$ the formal system obtained from $\mathbf{L J P}$ by allowing only intuitionistic sequents.

Proposition 3.1. For any intuitionistic sequent $\Gamma \rightarrow \Delta, \vdash \Gamma \rightarrow \Delta$ in $\mathbf{L J P}$ iff $\vdash \Gamma \rightarrow \Delta$ in $\mathbf{L} \mathbf{J} \mathbf{P}^{\prime}$.

Proof. (1) if part: obvious.

(2) only if part: Prove that for any sequent $\Gamma \rightarrow \Delta$, if $\vdash \Gamma \rightarrow \Delta$ in $\mathbf{L J P}$, then $\vdash \Gamma \rightarrow B_{1} \bigvee \cdots \bigvee B_{m}$ in $\mathbf{L} \mathbf{J P} \mathbf{P}^{\prime}$, where $\Delta=\left\{B_{1}, \cdots, B_{m}\right\}$.

Proposition 3.2 (Soundness Theorem of $\mathbb{L} \mathbb{J P}$ ). For any sequent $\Gamma \rightarrow \Delta$, if $\vdash \Gamma \rightarrow \Delta$ in $\mathbf{L J P}$, then $\models \Gamma \rightarrow \Delta$.

Proof. By induction on a proof of $\Gamma \rightarrow \Delta$.

\section{§4. Completeness}

The main purpose of this section is to establish the following theorem.

Theorem 4. 1 (Completeness Theorem for $\mathbf{L J P}$ ). Any consistent 
sequent $\Gamma \rightarrow \Delta$ is realizable.

A finite set $\Phi$ of formulae is called closed if it satisfies the following conditions :

(1) If $(A \wedge B) \in \Phi$, then $A \in \Phi$ and $B \in \Phi$.

(2) If $(A \vee B) \in \Phi$, then $A \in \Phi$ and $B \in \Phi$.

(3) If $\neg A \in \Phi$, then $A \in \Phi$.

(4) If $(A \supset B) \in \Phi$, then $A \in \Phi$ and $B \in \Phi$.

(5) If $[\alpha] A \in \Phi$, then $A \in \Phi$.

(6) If $[\alpha ; \beta] A \in \Phi$, then $[\alpha][\beta] A \in \Phi$.

(7) If $[\alpha \cup \beta] A \in \Phi$, then $[\alpha] A \in \Phi$ and $[\beta] A \in \Phi$.

(8) If $\left[\alpha^{*}\right] A \in \Phi$, then $[\alpha]\left[\alpha^{*}\right] A \in \Phi$.

(9) If $[A$ ? $] B \in \Phi$, then $A \in \Phi$ and $B \in \Phi$.

In the rest of this section we fix such a closed set, say, $\Phi$. A sequent $\Gamma \rightarrow \Delta$ is called $\Phi$-saturated if it satisfies the following conditions:

(1) $\Gamma \rightarrow \Delta$ is consistent.

(2) $\Gamma \cup \Delta=\Phi$.

It is easy to see that for any $\Phi$-saturated sequent $\Gamma \rightarrow \Delta, \Gamma \cap \Delta=\varnothing$.

Lemma 4. 2. Any consistent sequent $\Gamma \rightarrow \Delta$ can be extended to some consistent sequent $\widetilde{\Gamma} \rightarrow \tilde{\Delta}$ such that $\Phi \subseteq \widetilde{\Gamma} \cup \tilde{\Delta}$.

Corollary 4. 3. Any consistent sequent $\Gamma \rightarrow \Delta$, where $\Gamma \cup \Delta \subseteq \Phi$, can be extended to some $\Phi$-saturated sequent.

Now we define the $\Phi$-canonical Structure $\Omega(\Phi)=(S, \leqq, \rho, \pi)$ as follows:

(1) $S=\{\Gamma \rightarrow \Delta \mid \Gamma \rightarrow \Delta$ is $\Phi$-saturated $\}$.

(2) $\quad\left(\Gamma_{1} \rightarrow \Delta_{1}\right) \leqq\left(\Gamma_{2} \rightarrow \Delta_{2}\right)$ iff $\Gamma_{1} \subseteq \Gamma_{2}$.

(3) $\rho(a)=\left\{\left(\Gamma_{1} \rightarrow \Delta_{1}, \Gamma_{2} \rightarrow \Delta_{2}\right) \in S \times S \mid\left\{A \mid[a] A \in \Gamma_{1}\right\} \subseteq \Gamma_{2}\right\}$ 
for each atomic program $a$.

(4) $\pi(\Gamma \rightarrow \Delta, p)=1$ iff $p \in \Gamma$ for each atomic proposition $p$.

It is easy to see that $\Omega(\Phi)$ satisfies the conditions of the definition of a structure. The rest of this section is devoted almost completely to the proof of the following theorem, from which Theorem 4.1 follows at once.

Theorem 4. 4 (Fundamental Theorem of $\Omega(\Phi)$ ). For any formula $A \in \Phi$ and any sequent $\Gamma \rightarrow \Delta$ of $S, \pi(\Gamma \rightarrow \Delta, A)=1$ if $A \in \Gamma$ and $\pi(\Gamma \rightarrow \Delta, A)=0$ if $A \in \Delta$.

We define a notion of the test degree of a program $\alpha$ and a formula $A$, denoted by $t d(\alpha)$ and $t d(A)$ respectively, by simultaneous induction as follows:

(1) $t d(a)=t d(p)=0$ for any atomic program $a$ and atomic proposition $p$.

(2) $\quad t d(A \wedge B)=t d(A \bigvee B)=t d(A \supset B)=\max \{t d(A), t d(B)\}$.

(3) $\quad \operatorname{td}(\rceil A)=t d(A)$.

(4) $t d([\alpha] A)=\max \{t d(\alpha), t d(A)\}$.

(5) $\quad t d(\alpha ; \beta)=t d(\alpha \cup \beta)=\max \{t d(\alpha), t d(\beta)\}$.

(6) $\quad t d\left(\alpha^{*}\right)=t d(\alpha)$.

(7) $\quad t d(A$ ?) $=t d(A)+1$.

Our strategy of the proof of Theorem 4.4 is to prove the following theorem by induction on $i$.

Theorem 4.4 (i). For any sequent $\Gamma \rightarrow \Delta$ of $S$ and any formula $A \in \Phi$ such that $t d(A)<i, \pi(\Gamma \rightarrow \Delta, A)=1$ if $A \in \Gamma$ and $\pi(\Gamma \rightarrow \Delta, A)=0$ if $A \in \Delta$.

It is obvious that Theorem 4.4 (0) holds vacuously. Hence what we have to do is to prove Theorem $4.4(i+1)$, assuming Theorem 4.4 (i). To do it smoothly, we need several auxiliary notions and lemmas. We define the notions of the characteristic formula $\psi(\Gamma \rightarrow \Delta)$ of a 
sequent $\Gamma \rightarrow \Delta$ and of the characteristic formula $\psi(X)$ of a finite set $X$ of sequents as follows:

(1) $\psi(\Gamma \rightarrow \Delta)=A_{1} \wedge \cdots \wedge A_{n}$, where $\Gamma=\left\{A_{1}, \cdots, A_{n}\right\}$.

(2) $\psi(X)=\psi\left(\Gamma_{1} \rightarrow \Delta_{1}\right) \bigvee \cdots \vee \psi\left(\Gamma_{k} \rightarrow \Gamma_{k}\right)$, where

$$
X=\left\{\Gamma_{1} \rightarrow \Delta_{1}, \cdots, \Gamma_{k} \rightarrow \Delta_{k}\right\} .
$$

For any $Y \subseteq S$ and any program $\alpha$, the reakest precondition of $\alpha$ with respect to $Y$, denoted $w p(\alpha, Y)$, is defined as follows:

$$
w p(\alpha, Y)=\{s \in S \mid(s, t) \in \rho(\alpha) \text { implies } t \in Y \text { for any } t \in S\}
$$

For any $X, Y \subseteq S$ and any program $\alpha$, we say that $\alpha$ is partially correct with respect to precondition $X$ and postcondition $Y$ (notation: $\{X\} \alpha\{Y\})$ if $X \subseteq w p(\alpha, Y)$

Lemma 4.5 $(i+1)$. For any $X, Y \subseteq S$ and any program $\alpha$ such that $t d(\alpha)<i+1$, if $\{X\} \alpha\{Y\}$, then

$$
\vdash \psi(X) \rightarrow[\alpha] \psi(Y)
$$

Proof. The proof is carried out by induction on $\alpha$. Here we deal only with the following three critical cases.

(1) $\alpha$ is an atomic program, say, $a$ :

Let $X=\left\{\Gamma_{j} \rightarrow \Delta_{j} \mid 1 \leqq j \leqq n\right\}$. We assume, for the sake of simplicity, that $n=2$.

Suppose, for the sake of contradiction, that the sequent $\psi\left(\Gamma_{1} \rightarrow \Delta_{1}\right)$ $\rightarrow[a] \psi(Y)$ is consistent, which implies that the sequent $\Gamma_{1} \rightarrow[a] \psi(Y)$ is also consistent. So the sequent $\left\{A \mid[a] A \in \Gamma_{1}\right\} \rightarrow \psi(Y)$ is also consistent, for otherwise $\Gamma_{1} \rightarrow[a] \psi(Y)$ would be provable by rules $(\rightarrow[])$ and (extension). By Lemma 4.2, the sequent $\left\{A \mid[a] A \in \Gamma_{1}\right\} \rightarrow \psi(Y)$ can be extended to some consistent sequent $\widetilde{\Gamma} \rightarrow \tilde{\Delta}$ such that $\Phi \subseteq \widetilde{\Gamma} \cup \tilde{\Delta}$. Then it is easy to see that $\left(\Gamma_{1} \rightarrow \Delta_{1}, \widetilde{\Gamma} \cap \Phi \rightarrow \tilde{\Delta} \cap \Phi\right) \in \rho(a)$. Since $\left\{\Gamma_{1} \rightarrow \Delta_{1}\right\} a\{Y\}$ by assumption, $(\widetilde{\Gamma} \cap \Phi \rightarrow \tilde{\Delta} \cap \Phi) \in Y$. Hence

$$
\vdash \psi(\widetilde{\Gamma} \cap \Phi \rightarrow \tilde{\Delta} \cap \Phi) \rightarrow \psi(Y)
$$


This implies that

$$
\vdash \widetilde{\Gamma} \cap \Phi \rightarrow \psi(Y) .
$$

This contradicts the assumption that the sequent $\widetilde{\Gamma} \rightarrow \tilde{\Delta}$ is consistent and $\psi(Y) \in \tilde{\Delta}$. Thus we can conclude that

$$
\vdash \psi\left(\Gamma_{1} \rightarrow \Delta_{1}\right) \rightarrow[a] \psi(Y) .
$$

A similar argument shows that

$$
\vdash \psi\left(\Gamma_{2} \rightarrow \Delta_{2}\right) \rightarrow[a] \psi(Y) .
$$

By using rule $(\mathrm{V} \rightarrow)$ we can deduce from $(\mathrm{C})$ and (D) that

$$
\vdash \psi\left(\Gamma_{1} \rightarrow \Delta_{1}\right) \bigvee \psi\left(\Gamma_{2} \rightarrow \Delta_{2}\right) \rightarrow[a] \psi(Y),
$$

which was to be proved.

(2) $\alpha$ is of the form $\mathrm{A}$ ?:

Let $X=\left\{\Gamma_{j} \rightarrow \Delta_{j} \mid 1 \leqq j \leqq n\right\}$. We assume, for the sake of simplicity, that $n=2$. Suppose, for the sake of contradiction, that the sequent $\psi\left(\Gamma_{1}\right.$ $\left.\rightarrow \Gamma_{1}\right) \rightarrow[A$ ? $] \psi(Y)$ is consistent, which implies that the sequent $\Gamma_{1} \rightarrow[A$ ?] $\psi(Y)$ is also consistent. Hence the sequent $A, \Gamma_{1} \rightarrow \psi(Y)$ is also consistent, for otherwise the sequent $\Gamma_{1} \rightarrow[A$ ? $] \psi(Y)$ would be provable by rule $(\rightarrow[?])$. By Lemma 4.2 , the sequent $A, \Gamma_{1} \rightarrow \psi(Y)$ can be extended to some consistent sequent $\widetilde{\Gamma} \rightarrow \tilde{\Delta}$ such that $\Phi \subseteq \widetilde{\Gamma} \cup \tilde{\Delta}$. Since $t d(A)<i, \pi(\widetilde{\Gamma} \cap \Phi \rightarrow \tilde{\Delta} \cap \widetilde{\Phi}, A)=1$ by Theorem 4.4 (i). Since $\Gamma_{1} \subseteq \widetilde{\Gamma}$, $\left(\Gamma_{1} \rightarrow \Delta_{1}\right) \leqq(\widetilde{\Gamma} \cap \Phi \rightarrow \tilde{\Delta} \cap \Phi)$. Therefore $(\widetilde{\Gamma} \cap \Phi \rightarrow \tilde{\Delta} \cap \Phi) \in Y$. Hence

$$
\vdash \psi(\widetilde{\Gamma} \cap \Phi \rightarrow \tilde{\Delta} \cap \Phi) \rightarrow \psi(Y)
$$

This implies that

$$
\vdash \widetilde{\Gamma} \cap \Phi \rightarrow \psi(Y)
$$

This contradicts the assumption that the sequent $\widetilde{\Gamma} \rightarrow \tilde{\Delta}$ is consistent and $\psi(Y) \in \tilde{\Delta}$. Thus we can conclude that

$$
\vdash \psi\left(\Gamma_{1} \rightarrow \Delta_{1}\right) \rightarrow[A ?] \psi(Y) .
$$

Similarly,

$$
\vdash \psi\left(\Gamma_{2} \rightarrow \Delta_{2}\right) \rightarrow[A ?] \psi(Y) .
$$

By using rule $(\bigvee \rightarrow)$, we can deduce from $(C)$ and (D) that 


$$
\psi\left(\Gamma_{1} \rightarrow \Gamma_{1}\right) \bigvee \psi\left(\Gamma_{2} \rightarrow \Delta_{2}\right) \rightarrow[A ?] \psi(Y),
$$

which was to be proved.

(3) $\alpha$ is of the form $\beta^{*}$ :

Since $X \subseteq w p\left(\beta^{*}, Y\right)$ by Assumption,

$$
\vdash \psi(X) \rightarrow \psi\left(w p\left(\beta^{*}, Y\right)\right) .
$$

Since $\left\{w p\left(\beta^{*}, Y\right)\right\} \beta\left\{w p\left(\beta^{*}, Y\right)\right\}$,

$$
\vdash \psi\left(w p\left(\beta^{*}, Y\right)\right) \rightarrow[\beta] \psi\left(w p\left(\beta^{*}, Y\right)\right) .
$$

Hence by using rule $\left(\rightarrow\left[{ }^{*}\right]\right)$, we have that

$$
\vdash \psi\left(\omega p\left(\beta^{*}, Y\right)\right) \rightarrow\left[\beta^{*}\right] \psi\left(\omega p\left(\beta^{*}, Y\right)\right) .
$$

Since $\rho$ (true ?) $\subseteq \rho\left(\beta^{*}\right), w p\left(\beta^{*}, Y\right) \subseteq Y$.

Hence

$$
\vdash \psi\left(w p\left(\beta^{*}, Y\right)\right) \rightarrow \psi(Y) .
$$

By using rule $(\rightarrow[])$, we can deduce from (D) that

$$
\left[\beta^{*}\right] \psi\left(w p\left(\beta^{*}, Y\right)\right) \rightarrow\left[\beta^{*}\right] \psi(Y) .
$$

By using rule (cut) twice, we get from (A), (C) and (E) that

$$
\vdash \psi(X) \rightarrow\left[\beta^{*}\right] \psi(Y) \text {. }
$$

Lemma 4. $6(i+1)$. For any formula $A$ any program $\alpha$ and any sequents $\Gamma \rightarrow \Delta$ of $S$ such that $t d(\alpha)<i+1$, if $[\alpha] A \in \Delta$, then there exists a sequent $\Gamma^{\prime} \rightarrow \Delta^{\prime}$ of $S$ such that $\left(\Gamma \rightarrow \Delta, \Gamma^{\prime} \rightarrow \Delta^{\prime}\right) \in \rho(\alpha)$ and $A \in \Delta^{\prime}$.

Proof. Let $X=\{(\Pi \rightarrow \Sigma) \in S \mid A \in \Pi\}$. Suppose, for the sake of contradiction, that $\{\Gamma \rightarrow \Delta\} \alpha\{X\}$. Then by Lemma $4.5(i+1)$

$$
\vdash \psi(\Gamma \rightarrow \Delta) \rightarrow[\alpha] \psi(X) .
$$

It follows from the definition of $X$ that

$$
\vdash \psi(X) \rightarrow A
$$

By using rules (cut) and $(\rightarrow[])$, we can deduce from (A) and (B) that

$$
\vdash \psi(\Gamma \rightarrow \Delta) \rightarrow[\alpha] A
$$


It follows from (C) that

$$
\vdash \Gamma \rightarrow[\alpha] A,
$$

which contradicts the assumption that the sequent $\Gamma \rightarrow \Delta$ is consistent and $[\alpha] A \in \Delta$. This completes the proof.

Lemma 4.7 $(i+1)$. For any formula $A$, any program $\alpha$ and any sequents $\Gamma \rightarrow \Delta, \Gamma^{\prime} \rightarrow \Delta^{\prime}$ of $S$ such that $t d(\alpha)<i+1$ and $(\Gamma \rightarrow \Delta$, $\left.\Gamma^{\prime} \rightarrow \Delta^{\prime}\right) \in \rho(\alpha)$, if $[\alpha] A \in \Gamma$, then $A \in \Gamma^{\prime}$.

Proof. Similar to that of Lemma $4.5(i+1)$.

Now we are ready to complete the proof of Theorem $4.4(i+1)$.

Proof of Theorem 4.4 $(i+1)$. By induction on the construction of a formula $A \in \Phi$. Use Lemmas $4.6(i+1)$ and $4.7(i+1)$ in dealing with formulae of the form $[\alpha] A$.

We have completed the proof of Theorem 4.4. By combining Proposition 3.2 and Theorem 4.1, we have

Theorem 4. 8. For any sequent $\Gamma \rightarrow A$,

$$
\vdash \Gamma \rightarrow \Delta \quad \text { iff } \quad \models \Gamma \rightarrow \Delta
$$

The finite model property shown in Theorem 4.4 establishes

Theorem 4.9 (Decidability of LJP). $\mathbb{L} \mathbb{P P}$ is decidable.

\section{References}

[1] Fitting, M. C., Intuitionistic logic model theory and forcing, North-Holland, Amsterdam, 1969.

[2] Gabbay, D. M., Semantical investigations in Heyting's intuitionistic logic, D. Reidel, Dordrecht-Boston-London, 1981.

[3] Harel, D., First-order dynamic logic, Lecture Notes in Computer Science, 68 Springer, Berlin-Heidelberg-New York, 1979.

[4] Leivant, D., Proof theoretic methodology for propositional dynamic logic, in: Formalization of programming concepts, Lecture Notes in Computer Science, 107 
Springer, Berlin-Heidelberg-New York, (1981), 356-373.

[5] Nishimura, H., Sequential methods in propositional dynamic logic, Acta Informatica, 12 (1979), 377-400.

[6] Takeuti, G., Proof theory, North-Holland, Amsterdam, 1975. 